\title{
Liouville type theorem associated with the wave equation
}

\author{
KATSUNORI SHIMOMURA*
}

\section{Introduction}

The well-known Liouville's theorem states that every conformal mapping in the $n$-dimensional Euclidean space $(n \geqq 3)$ is a similarity or an inversion with respect to a sphere composed with similarities. The conformal mapping associates with the Laplace equation in the following sense. Let $D, E \subset \mathbb{R}^{n}$ be domains, $f: D \rightarrow E$ a nonconstant $C^{2}$-mapping, and $\varphi$ a positive $C^{2}$-function on $D$. Then the transformation $u \mapsto \varphi \cdot(u \circ f)$ for $C^{2}$-function $u$ on $D$ preserves the solutions of the Laplace equation on $E$ if only if $f$ is a conformal mapping.

In this note, we consider a Liouville type theorem associated with the wave equation and determine transformations which preserve the solutions of the wave equation. We note that Sugimoto considered this problem in [4] and obtained a partial result.

Let $\mathbb{R}^{n+1}$ be the $(n+1)$-dimensional Euclidean space $(n \geqq 2)$, and denote its point by $x=\left(x_{0}, x_{1}, \ldots, x_{n}\right)$. We introduce Lorentzian scalar product $\langle\cdot, \cdot\rangle$ on $\mathbb{R}^{n+1}$ defined by

$$
\langle x, y\rangle=-x_{0} y_{0}+x_{1} y_{1}+\cdots+x_{n} y_{n} .
$$

Let $D \subset \mathbb{R}^{n+1}, E \subset \mathbb{R}^{k}$ be domains and $f=\left(f_{0}, f_{1}, \ldots, f_{n}\right): D \rightarrow E$ a $C^{1}$ mapping. The first derivative $f^{\prime}(x)$ of $f$ at $x \in D$ is a linear mapping $\mathbb{R}^{n+1} \rightarrow \mathbb{R}^{k}$ such that

$$
f^{\prime}(x) u=\lim _{h \rightarrow 0} \frac{f(x+h u)-f(x)}{h}, \quad u \in \mathbb{R}^{n+1} .
$$

Definition 1.1. Let $D, E \subset \mathbb{R}^{n+1}$ be domains. A $C^{1}$-mapping $f: D \rightarrow E$ is said to be Lorentzian conformal if there exists a function $\lambda(x)=\lambda_{f}(x)>0$ defined on $D$ such that

$$
\left\langle f^{\prime}(x) u, f^{\prime}(x) v\right\rangle=\lambda(x)^{2}\langle u, v\rangle
$$

holds for all $x \in D$ and all $u, v \in \mathbb{R}^{n+1}$.

Received 13 May, 2011; revised 7 June, 2011

2000 Mathematics Subject Classification. 31B99, 35K99, 35A30

Key Words and Phrases. conformal mapping, semi-euclidean, wave equation

*Partially supported by Grant-in-aid for Scientific Research (C) No.19540161, Japan Society for the Promotion of Science.

*Ibaraki University, Mito, Ibaraki, 310-8512, Japan.(shimomur@mx.ibaraki.ac.jp) 
Let

$$
\mathrm{O}(1, n):=\left\{R \in \mathrm{GL}(n+1, \mathbb{R}) ;\langle R x, R y\rangle=\langle x, y\rangle \text { for all } x, y \in \mathbb{R}^{n+1}\right\} .
$$

Our Liouville type theorem is the following:

Theorem 1.1. Let $n \geqq 2$ and $f$ be a Lorentzian conformal $C^{4}$-mapping defined on a domain $D \subset \mathbb{R}^{n+1}$. Then $f$ has one of the following forms :

$$
\begin{aligned}
& f(x)=\frac{\alpha R(x-a)}{\langle x-a, x-a\rangle}+b, \\
& f(x)=\alpha R B(S(x-a))+b, \\
& f(x)=\alpha R x+b,
\end{aligned}
$$

where $\alpha>0, R, S \in \mathrm{O}(1, n), a, b \in \mathbb{R}^{n+1}$, and

$$
B(x)=\left(\frac{\langle x, x\rangle+1}{2\left(x_{0}+x_{1}\right)}, \frac{\langle x, x\rangle-1}{2\left(x_{0}+x_{1}\right)}, \frac{x_{2}}{x_{0}+x_{1}}, \ldots, \frac{x_{n}}{x_{0}+x_{1}}\right) .
$$

Next we consider the wave equation

$$
W u:=\frac{\partial^{2} u}{\partial x_{0}^{2}}-\sum_{j=1}^{n} \frac{\partial^{2} u}{\partial x_{j}^{2}}=0
$$

on $\mathbb{R}^{n+1}$ and transformations which preserve the solutions of the wave equation.

Definition 1.2. Let $D \subset \mathbb{R}^{n+1}$ be a domain, $f: D \rightarrow \mathbb{R}^{n+1}$ a $C^{2}$-mapping, and $\varphi$ a positive $C^{2}$-function on $D$. A pair $(f, \varphi)$ is called a transformation which preserves the wave equation, if $f$ and $\varphi$ satisfy the following conditions:

(1) $f^{\prime}(x)$ is non-degenerate for all $x \in D$.

(2) For every solution $u$ of the wave equation on $\mathbb{R}^{n+1}, \varphi \cdot(u \circ f)$ satisfies the wave equation on $D$.

By Theorem 1.1, we can determine the transformation which preserves the wave equation.

Theorem 1.2. Let $n \geqq 2, f$ be a $C^{4}$-mapping, and $\varphi$ a positive function defined on a domain $D \subset \mathbb{R}^{n+1}$. If $(f, \varphi)$ be a transformation which preserve the wave equation, then $f$ and $\varphi$ have one of the following forms :

$$
\begin{array}{llrl}
f(x) & =\frac{\alpha R(x-a)}{\langle x-a, x-a\rangle}+b, & \varphi(x) & =C|\langle x-a, x-a\rangle|^{\frac{1-n}{2}}, \\
f(x) & =\alpha R B(S(x-a))+b, & \varphi(x) & =C|\langle v, S(x-a)\rangle|^{\frac{1-n}{2}}, \\
f(x) & =\alpha R x+b, & \varphi(x) & =C,
\end{array}
$$

where $\alpha>0, R, S \in \mathrm{O}(1, n), a, b \in \mathbb{R}^{n+1}$, and

$$
B(x)=\left(\frac{\langle x, x\rangle+1}{2\left(x_{0}+x_{1}\right)}, \frac{\langle x, x\rangle-1}{2\left(x_{0}+x_{1}\right)}, \frac{x_{2}}{x_{0}+x_{1}}, \ldots, \frac{x_{n}}{x_{0}+x_{1}}\right),
$$

and $v=(-1,1,0, \ldots, 0) \in \mathbb{R}^{n+1}$. 


\section{Lorentzian space and Lorentzian conformal mapping}

In the last section, we introduced Lorentzian scalar product. Let $J$ be the $(n+$ $1) \times(n+1)$ diagonal matrix such that

$$
J=\operatorname{diag}(-1,1, \ldots, 1) .
$$

Then the Lorentzian scalar product $\langle\cdot, \cdot\rangle$ is written by the Euclidean scalar product $(\cdot, \cdot)$ as

$$
\langle x, y\rangle=(J x, y)=(x, J y), \quad x, y \in \mathbb{R}^{n+1} .
$$

The vector space $\mathbb{R}^{n+1}$ with this Lorentzian scalar product is called Lorentzian $(n+1)$ space, and is denoted by $\mathbb{R}^{1, n}$.

Recall that $\mathrm{O}(1, n):=\left\{R \in \mathrm{GL}(n+1, \mathbb{R}) ;\langle R x, R y\rangle=\langle x, y\rangle\right.$ for all $\left.x, y \in \mathbb{R}^{n+1}\right\}$. $\mathrm{O}(1, n)$ is the group of all matrices which preserve the Lorentzian scalar product. It is clear that $R \in \mathrm{O}(1, n)$ is equivalent to ${ }^{t} R J R=J$. We also remark that $R \in \mathrm{O}(1, n)$ if and only if $J=R J^{t} R$ by taking the inverse.

For a subspace $X$ of $\mathbb{R}^{1, n}$, the Lorentzian complement of $X$ is defined by

$$
X^{L}=\left\{x \in \mathbb{R}^{1, n} ;\langle x, y\rangle=0, y \in X\right\} .
$$

The following Lemmas will be used in section 3 .

Lemma 2.1. For any subspace $X$ of $\mathbb{R}^{1, n}, X^{L}=(J X)^{\perp}=J\left(X^{\perp}\right)$ and $\left(X^{L}\right)^{L}=X$ hold, where $(\cdot)^{\perp}$ denotes the orthogonal complement.

Proof. Since $\langle x, y\rangle=0$ is equivalent to $(x, J y)=0, x \in X^{L}$ is equivalent to $x \in$ $(J X)^{\perp}$. Since $\langle x, y\rangle=0$ is also equivalent to $(J x, y)=0$ and $J^{2}$ is equal to the unit matrix, $x \in X^{L}$ is equivalent to $x \in J\left(X^{\perp}\right)$. Therefore $X^{L}=(J X)^{\perp}=J\left(X^{\perp}\right)$ holds. The latter statement follows from $\left(X^{L}\right)^{L}=J\left(\left((J X)^{\perp}\right)^{\perp}\right)=J^{2} X=X$.

Lemma 2.2. Let $X$ be a subspace of $\mathbb{R}^{1, n}, \alpha \neq 0$, and $R \in \mathrm{O}(1, n)$. Then $\alpha R\left(X^{L}\right)=$ $(\alpha R X)^{L}$ holds.

Proof. $x \in \alpha R\left(X^{L}\right)$ if and only if $\left\langle\alpha^{-1} R^{-1} x, y\right\rangle=0$ for all $y \in X$. Then

$$
\left\langle\alpha \alpha^{-1} R R^{-1} x, \alpha R y\right\rangle=\langle x, \alpha R y\rangle=0
$$

for all $y \in X$. This means $x \in(\alpha R X)^{L}$.

The following proposition follows easily from the chain rule.

Proposition 2.1. (1) If $f: D \rightarrow E$ and $g: E \rightarrow F$ are Lorentzian conformal mappings, then the composition mapping $g \circ f: D \rightarrow F$ is also a Lorentzian conformal mapping, and

$$
\lambda_{g \circ f}(x)=\lambda_{g}(f(x)) \lambda_{f}(x), \quad x \in D
$$

holds. 
(2) If $f: D \rightarrow E$ is a Lorentzian conformal mapping, then $f$ has local inverse $f^{-1}$ at each point of $f(D), f^{-1}$ is also Lorentzian conformal, and

$$
\lambda_{f^{-1}}(f(x))=\frac{1}{\lambda_{f}(x)}
$$

holds.

In the following, we list fundamental Lorentzian conformal mappings.

Example 2.1 (Lorentzian similarity). The mapping

$$
f(x)=\alpha R x+a \quad\left(\alpha \in \mathbb{R}, \alpha>0, R \in \mathrm{O}(1, n), a \in \mathbb{R}^{1, n}\right)
$$

is a Lorentzian conformal mapping defined on $\mathbb{R}^{1, n}$ satisfying $\lambda_{f}(x)=\alpha$.

Example 2.2 (Lorentzian inversion). The mapping

$$
K(x)=\frac{x}{\langle x, x\rangle}
$$

is a Lorentzian conformal mapping defined on each connected component of $\{x \in$ $\left.\mathbb{R}^{1, n} ;\langle x, x\rangle \neq 0\right\}$. We call $K$ inversion (mapping). By simple calculation, we have

$$
K^{-1}=K
$$

and

$$
\lambda_{K}(x)=\frac{1}{|\langle x, x\rangle|} .
$$

Example 2.3 (Bateman mapping). The mapping

$$
B(x)=\left(\frac{\langle x, x\rangle+1}{2\left(x_{0}+x_{1}\right)}, \frac{\langle x, x\rangle-1}{2\left(x_{0}+x_{1}\right)}, \frac{x_{2}}{x_{0}+x_{1}}, \ldots, \frac{x_{n}}{x_{0}+x_{1}}\right),
$$

is a Lorentzian conformal mapping defined on each connected component half space of $\left\{x \in \mathbb{R}^{1, n} ; x_{0}+x_{1} \neq 0\right\}$. We call $B$ the mapping of the Bateman transformation. By easy calculation, we have

$$
B^{-1}(x)=\left(\frac{\langle x, x\rangle+1}{2\left(x_{0}-x_{1}\right)}, \frac{1-\langle x, x\rangle}{2\left(x_{0}-x_{1}\right)}, \frac{x_{2}}{x_{0}-x_{1}}, \ldots, \frac{x_{n}}{x_{0}-x_{1}}\right),
$$

and

$$
\lambda_{B}(x)=\frac{1}{\left|x_{0}+x_{1}\right|}, \quad \lambda_{B^{-1}}(x)=\frac{1}{\left|x_{0}-x_{1}\right|} .
$$

\section{Liouville type theorem for Lorentzian conformal mapping}

In this section, we shall prove Theorem 1.1. The former part follows from similar arguments in the proof of Liouville's theorem for conformal mapping in [1]. 
The second derivative of a $C^{2}$-mapping $f: \mathbb{R}^{n+1} \rightarrow \mathbb{R}^{k}$ is a bilinear mapping $f^{\prime \prime}(x): \mathbb{R}^{n+1} \times \mathbb{R}^{n+1} \rightarrow \mathbb{R}^{k}$ such that

$$
f^{\prime \prime}(x)[u, v]=\lim _{h \rightarrow 0} \frac{1}{h}\left(f^{\prime}(x+h u) v-f^{\prime}(x) v\right) .
$$

The third derivative of a $C^{3}$-mapping $f: \mathbb{R}^{n+1} \rightarrow \mathbb{R}^{k}$ is a trilinear mapping $f^{\prime \prime \prime}(x)$ : $\mathbb{R}^{n+1} \times \mathbb{R}^{n+1} \times \mathbb{R}^{n+1} \rightarrow \mathbb{R}^{k}$ such that

$$
f^{\prime \prime \prime}(x)[u, v, w]=\lim _{h \rightarrow 0} \frac{1}{h}\left(f^{\prime \prime}(x+h u)[v, w]-f^{\prime \prime}(x)[v, w]\right) .
$$

Lemma 3.1. Let $f$ be a $C^{2}$-mapping defined on a domain $D \subset \mathbb{R}^{1, n}$. If $f$ is a Lorentzian conformal mapping, then for each $u, v, w \in \mathbb{R}^{1, n}$,

$$
\left\langle f^{\prime \prime}(x)[u, v], f^{\prime}(x) w\right\rangle+\left\langle f^{\prime \prime}(x)[u, w], f^{\prime}(x) v\right\rangle=2 \lambda(x)\langle v, w\rangle \lambda^{\prime}(x) u
$$

holds.

Proof. Taking the derivative in $u$ direction of the Lorentzian conformality condition (1.1)

$$
\left\langle f^{\prime}(x) v, f^{\prime}(x) w\right\rangle=\lambda(x)^{2}\langle v, w\rangle
$$

we have (3.1).

Lemma 3.2. Let $f$ be a $C^{2}$-mapping defined on a domain $D \subset \mathbb{R}^{1, n}$. If $f$ is a Lorentzian conformal mapping and $\langle u, v\rangle=\langle v, w\rangle=\langle w, u\rangle=0$, then

$$
\left\langle f^{\prime \prime}(x)[u, v], f^{\prime}(x) w\right\rangle=0 .
$$

Proof. We put $A[u, v, w]=\left\langle f^{\prime \prime}(x)[u, v], f^{\prime}(x) w\right\rangle$ for $u, v, w \in \mathbb{R}^{1, n}$. Then $A$ satisfies

$$
\begin{aligned}
& A[v, u, w]=A[u, v, w], \\
& A[u, v, w]=-A[u, w, v] \text { if }\langle v, w\rangle=0 .
\end{aligned}
$$

In fact (3.2) is clear from the symmetry of the bilinear map $f^{\prime \prime}(x)$, and (3.3) follows from Lemma 3.1 immediately.

Since $\langle u, v\rangle=\langle v, w\rangle=\langle w, u\rangle=0$, then we have $A[u, v, w]=A[v, u, w]=$ $-A[v, w, u]=-A[w, v, u]=A[w, u, v]=A[u, w, v]=-A[u, v, w]$ by (3.2) and (3.3). Therefore $A[u, v, w]=0$. This proves the lemma.

Lemma 3.3. Let $f$ be a Lorentzian conformal $C^{2}$-mapping defined on a domain $D \subset \mathbb{R}^{1, n}$. If $\lambda$ is equal to a constant $\alpha$ on $D$, then $f$ is a similarity of form $f(x)=$ $\alpha R x+a$, with some $R \in \mathrm{O}(1, n)$ and $a \in \mathbb{R}^{1, n}$.

Proof. Since $\lambda$ is constant, $\left\langle f^{\prime \prime}(x)[u, v], f^{\prime}(x) w\right\rangle+\left\langle f^{\prime \prime}(x)[u, w], f^{\prime}(x) v\right\rangle=0$ for all $u, v, w \in \mathbb{R}^{1, n}$ by Lemma 3.1. Then $A[u, v, w]=\left\langle f^{\prime \prime}(x)[u, v], f^{\prime}(x) w\right\rangle$ satisfies (3.2) and

$$
A[u, v, w]=-A[u, w, v] \quad\left(u, v, w \in \mathbb{R}^{1, n}\right) .
$$


The same argument as in the proof of Lemma 3.2, $A[u, v, w]=0$ follows for all $u, v, w \in$ $\mathbb{R}^{1, n}$. Since $f^{\prime}(x)$ is surjective, $f^{\prime \prime}(x)[u, v]=0$ for all $u, v \in \mathbb{R}^{1, n}$, and hence $f^{\prime \prime}(x)=0$. Then $\alpha^{-1} f^{\prime}(x)$ is a constant matrix $R$ satisfying $\langle R u, R v\rangle=\langle u, v\rangle$. Therefore $R \in$ $\mathrm{O}(1, n)$ and $f(x)=\alpha R x+a$, where $a \in \mathbb{R}^{1, n}$.

The following lemma is well-known in linear algebra.

Lemma 3.4. Let $l(u, v)$ is a symmetric bilinear form on $\mathbb{R}^{1, n}$. If $l(u, v)=0$ for all $u, v \in \mathbb{R}^{1, n}$ with $\langle u, v\rangle=0$, then there exists a constant $c \in \mathbb{R}$ such that

$$
l(u, v)=c\langle u, v\rangle \quad\left(u, v \in \mathbb{R}^{1, n}\right) .
$$

Lemma 3.5. Let $f$ be a $C^{2}$-mapping defined on a domain $D$. If $f$ is a Lorentzian conformal mapping and $\langle u, v\rangle=0$, then for each $x \in D$,

$$
f^{\prime \prime}(x)[u, v]=\lambda(x)^{-1} \lambda^{\prime}(x) v f^{\prime}(x) u+\lambda(x)^{-1} \lambda^{\prime}(x) u f^{\prime}(x) v .
$$

Proof. Assume $\langle u, u\rangle \neq 0$ and $\langle v, v\rangle \neq 0$. If $\langle u, v\rangle=0$, then Lemma 3.2 implies that for any $w \in(\mathbb{R} u+\mathbb{R} v)^{L}$,

$$
\left\langle f^{\prime \prime}(x)[u, v], f^{\prime}(x) w\right\rangle=0 .
$$

Hence $f^{\prime \prime}(x)[u, v] \in\left(f^{\prime}(x)\left((\mathbb{R} u+\mathbb{R} v)^{L}\right)\right)^{L}$. By Lemma 2.1 and 2.2, we obtain

$\left(f^{\prime}(x)\left((\mathbb{R} u+\mathbb{R} v)^{L}\right)\right)^{L}=f^{\prime}(x)\left(\left((\mathbb{R} u+\mathbb{R} v)^{L}\right)^{L}\right)=f^{\prime}(x)(\mathbb{R} u+\mathbb{R} v)=\mathbb{R} f^{\prime}(x) u+\mathbb{R} f^{\prime}(x) v$.

Therefore there exist constants $c_{1}, c_{2} \in \mathbb{R}$ such that

$$
f^{\prime \prime}(x)[u, v]=c_{1} f^{\prime}(x) u+c_{2} f^{\prime}(x) v .
$$

Applying Lemma 3.1 with $w=v$, we have

$$
\left\langle f^{\prime \prime}(x)[u, v], f^{\prime}(x) v\right\rangle=\lambda(x)\langle v, v\rangle \lambda^{\prime}(x) u
$$

and

$$
c_{1}\left\langle f^{\prime}(x) u, f^{\prime}(x) v\right\rangle+\left\langle c_{2} f^{\prime}(x) v, f^{\prime}(x) v\right\rangle=\lambda(x)\langle v, v\rangle \lambda^{\prime}(x) u .
$$

Since $\langle v, u\rangle=0$ and $f$ is Lorentzian conformal, we have

$$
c_{2} \lambda(x)^{2}\langle v, v\rangle=\lambda(x)\langle v, v\rangle \lambda^{\prime}(x) u,
$$

and hence $c_{2}=\lambda(x)^{-1} \lambda^{\prime}(x) u$, because $\langle v, v\rangle \neq 0$. Similarly, we have $c_{1}=\lambda(x)^{-1} \lambda^{\prime}(x) v$. Then we have (3.5) for all $u, v$ with $\langle u, v\rangle=0$ by continuity.

Proposition 3.1. Let $f$ be Lorentzian conformal and put $\rho(x)=1 / \lambda(x)$. If $f$ is of $C^{4}$, then there exists a constant $c$ such that for every $u, v \in \mathbb{R}^{1, n}$,

$$
\rho^{\prime \prime}(x)[u, v]=c\langle u, v\rangle .
$$


Proof. Let $u, v \in \mathbb{R}^{1, n}$ satisfy $\langle u, v\rangle=0,\langle u, u\rangle \neq 0$ and $\langle v, v\rangle \neq 0$. Then by Proposition 3.5 ,

$$
f^{\prime \prime}(x)[u, v]=\lambda(x)^{-1} \lambda^{\prime}(x) v f^{\prime}(x) u+\lambda(x)^{-1} \lambda^{\prime}(x) u f^{\prime}(x) v .
$$

Then we have

$$
\rho(x) f^{\prime \prime}(x)[u, v]+\rho^{\prime}(x) v f^{\prime}(x) u+\rho^{\prime}(x) u f^{\prime}(x) v=0 .
$$

Since $n \geqq 2$, we can take $w \in \mathbb{R}^{1, n}$ such that $\langle w, w\rangle \neq 0$ and $\langle u, w\rangle=\langle v, w\rangle=0$. Taking the derivative in direction $w$, we have

$$
\begin{aligned}
& \rho^{\prime}(x) w f^{\prime \prime}(x)[u, v]+\rho(x) f^{\prime \prime \prime}(x)[u, v, w]+\rho^{\prime \prime}(x)[v, w] f^{\prime}(x) u \\
& \quad+\rho^{\prime}(x) v f^{\prime \prime}(x)[u, w]+\rho^{\prime \prime}(x)[u, w] f^{\prime}(x) v+\rho^{\prime}(x) u f^{\prime \prime}(x)[v, w]=0 .
\end{aligned}
$$

Assume $\langle v, w\rangle=0$ and interchange $u$ and $w$ in the equation. Then

$$
\begin{aligned}
& \rho^{\prime}(x) u f^{\prime \prime}(x)[w, v]+\rho(x) f^{\prime \prime \prime}(x)[w, v, u]+\rho^{\prime \prime}(x)[v, u] f^{\prime}(x) w \\
& \quad+\rho^{\prime}(x) v f^{\prime \prime}(x)[w, u]+\rho^{\prime \prime}(x)[w, u] f^{\prime}(x) v+\rho^{\prime}(x) w f^{\prime \prime}(x)[v, u]=0 .
\end{aligned}
$$

Subtract (3.7) from (3.6). Then we have

$$
\rho^{\prime \prime}(x)[v, w] f^{\prime}(x) u-\rho^{\prime \prime}(x)[v, u] f^{\prime}(x) w=0,
$$

and hence

$$
\begin{aligned}
\rho^{\prime \prime}(x)[v, w] \lambda(x)^{2}\langle u, w\rangle-\rho^{\prime \prime}(x)[v, u] \lambda(x)^{2}\langle w, w\rangle \\
\quad=\rho^{\prime \prime}(x)[v, w]\left\langle f^{\prime}(x) u, f^{\prime}(x) w\right\rangle-\rho^{\prime \prime}(x)[v, u]\left\langle f^{\prime}(x) w, f^{\prime}(x) w\right\rangle=0 .
\end{aligned}
$$

Since $\langle u, w\rangle=0$ and $\langle w, w\rangle \neq 0$, we obtain

$$
\rho^{\prime \prime}(x)[u, v]=0,
$$

for all $u, v \in \mathbb{R}^{1, n}$ with $\langle u, v\rangle=0,\langle u, u\rangle \neq 0$ and $\langle v, v\rangle \neq 0$. By continuity, we can drop $\langle u, u\rangle \neq 0$ and $\langle v, v\rangle \neq 0$. By Lemma 3.4,

$$
\rho^{\prime \prime}(x)[u, v]=c(x)\langle u, v\rangle,
$$

holds for all $u, v \in \mathbb{R}^{1, n}$ with some $c(x) \in \mathbb{R}$.

To show $c(x)$ is constant, take the derivative of (3.8) in direction $w$. We have

$$
\rho^{\prime \prime \prime}(x)[u, v, w]=c^{\prime}(x) w\langle u, v\rangle,
$$

and then

$$
\begin{aligned}
\left\langle\left(c^{\prime}(x) w\right) u-\left(c^{\prime}(x) u\right) w, v\right\rangle & =c^{\prime}(x) w\langle u, v\rangle-c^{\prime}(x) u\langle w, v\rangle \\
& =\rho^{\prime \prime \prime}(x)[u, v, w]-\rho^{\prime \prime \prime}(x)[w, v, u]=0,
\end{aligned}
$$

holds for all $u, v, w \in \mathbb{R}^{1, n}$. That implies

$$
\left(c^{\prime}(x) w\right) u-\left(c^{\prime}(x) u\right) w=0,
$$


and hence

$$
c^{\prime}(x) u=c^{\prime}(x) w=0
$$

for each linearly independent pair of $u$ and $w$. By continuity, we obtain $c^{\prime}(x)=0$. Thus we have the proposition.

Proposition 3.2. Let $f$ be a $C^{4}$-mapping defined on a domain D. If $f$ is a Lorentzian conformal mapping, then $\lambda_{f}(x)$ has one of the following forms :

$$
\begin{array}{ll}
\lambda_{f}(x)=\frac{\alpha}{\langle x-a, x-a\rangle}, & \text { with } \alpha \in \mathbb{R}, \alpha \neq 0, a \in \mathbb{R}^{1, n}, \\
\lambda_{f}(x)=\frac{\alpha}{\langle d, x-a\rangle}, & \text { with } \alpha \in \mathbb{R}, \alpha \neq 0, a, d \in \mathbb{R}^{1, n}, d_{0}=1,\langle d, d\rangle=0, \\
\lambda_{f}(x)=\alpha, & \text { with } \alpha \in \mathbb{R}, \alpha>0 .
\end{array}
$$

Proof. We may assume that $\rho(x)$ is not constant. Since $\rho^{\prime \prime}(x)[u, v]=c\langle u, v\rangle$ with some constant $c \in \mathbb{R}$ by Proposition 3.1,

$$
\rho(x)=\frac{c}{2}\langle x, x\rangle+c_{0} x_{0}+\cdots+c_{n} x_{n}+c_{n+1},
$$

where $c_{0}, \ldots, c_{n+1} \in \mathbb{R}$. If $c \neq 0$, then

$$
\lambda_{f}(x)=\frac{\alpha}{\langle x-a, x-a\rangle+\beta},
$$

where $\alpha, \beta \in \mathbb{R}, a \in \mathbb{R}^{1, n}$, with $\alpha \neq 0$. If $c=0$, then

$$
\lambda_{f}(x)=\frac{\alpha}{\langle d, x-a\rangle},
$$

where $\alpha \in \mathbb{R}, a, d \in \mathbb{R}^{1, n}$, with $\alpha \neq 0$ and $d_{0}=1$. We have only to show $\beta=0$ and $\langle d, d\rangle=0$.

Since the local inverse $f^{-1}$ is also a Lorentzian conformal mapping, $\lambda_{f^{-1}}$ has the form

$$
\lambda_{f^{-1}}(y)=\frac{\tilde{\alpha}}{\langle y-\tilde{a}, y-\tilde{a}\rangle+\tilde{\beta}} \quad \text { or } \quad \lambda_{f^{-1}}(y)=\frac{\tilde{\alpha}}{\langle\tilde{d}, y-\tilde{a}\rangle},
$$

where $\tilde{\alpha}, \tilde{\beta} \in \mathbb{R}, \tilde{a}, \tilde{d} \in \mathbb{R}^{1, n}$, with $\tilde{\alpha} \neq 0$ and $\tilde{d}_{0}=1$ by the same argument. Then $\left(\lambda_{f^{-1}}(f(x))\right)^{-1}=\lambda_{f}(x)$ implies that $f$ satisfies one of the following :

$$
\begin{array}{lll}
\langle f(x)-\tilde{a}, f(x)-\tilde{a}\rangle+\tilde{\beta}=\frac{\alpha_{0}}{\langle x-a, x-a\rangle+\beta}, & \lambda_{f}(x)=\frac{\alpha}{\langle x-a, x-a\rangle+\beta}, \\
\langle\tilde{d}, f(x)-\tilde{a}\rangle=\frac{\alpha_{0}}{\langle x-a, x-a\rangle+\beta}, & \lambda_{f}(x)=\frac{\alpha}{\langle x-a, x-a\rangle+\beta}, \\
\langle f(x)-\tilde{a}, f(x)-\tilde{a}\rangle+\tilde{\beta}=\frac{\alpha_{0}}{\langle d, x-a\rangle}, & \lambda_{f}(x)=\frac{\alpha}{\langle d, x-a\rangle}, \\
\langle\tilde{d}, f(x)-\tilde{a}\rangle=\frac{\alpha_{0}}{\langle d, x-a\rangle}, & \lambda_{f}(x)=\frac{\alpha}{\langle d, x-a\rangle},
\end{array}
$$


where $\alpha_{0}, \beta, \tilde{\beta} \in \mathbb{R}, a, \tilde{a}, d, \tilde{d} \in \mathbb{R}^{1, n}$ with $\alpha_{0} \neq 0, d_{0}=\tilde{d}_{0}=1$.

First we shall prove that the cases of (3.13) and (3.14) does not occur. In fact, equation (3.13) shows that for each $r \in \mathbb{R}, f$ maps hypersurfaces $S_{r}=\{x ;\langle x-a, x-$ $a\rangle+\beta=r\}$ intersecting $D$ into hyperplanes $P_{r}=\left\{y ;\langle\tilde{d}, y-\tilde{a}\rangle=\alpha_{0} / r\right\}$. Choose $v \in \mathbb{R}^{n+1}$ with $\langle v, v\rangle \neq 0$ such that the line $x(t)=t v+a(t \in \mathbb{R})$ intersects $D$. In general, $x(t)$ is Lorentz orthogonal to all $S_{r}(r \in \mathbb{R})$. Since $f$ is Lorentzian conformal, the image curve $f(x(t))$ of the line $x(t)$ is Lorentz orthogonal to all $P_{r}(r \in \mathbb{R})$ and hence $f(x(t))$ lies in the line $s \tilde{d}+\tilde{v}(s \in \mathbb{R})$ with some $\tilde{v} \in \mathbb{R}^{1, n}$. So there exists a $C^{4}$-function $s(t)$ such that $f(x(t))=s(t) \tilde{d}+\tilde{v}$. Differentiating the both sides of the equation by $t$, we have

$$
f^{\prime}(x(t)) v=s^{\prime}(t) \tilde{d}
$$

Then Lorentzian conformality of $f$ implies

$$
s^{\prime}(t)^{2}\langle\tilde{d}, \tilde{d}\rangle=\left\langle f^{\prime}(x(t)) v, f^{\prime}(x(t)) v\right\rangle=\lambda_{f}(x(t))^{2}\langle v, v\rangle=\frac{\alpha^{2}\langle v, v\rangle}{\left(\langle v, v\rangle t^{2}+\beta\right)^{2}}
$$

and hence

$$
s^{\prime}(t)=\frac{\alpha_{1}}{t^{2}+\beta_{1}}
$$

with some $\alpha_{1}, \beta_{1} \in \mathbb{R}, \alpha_{1} \neq 0$. On the other hand, substituting $f(x(t))=s(t) \tilde{d}+\tilde{v}$ and $x(t)=t v+a$ into (3.13), we have

$$
s(t)\langle\tilde{d}, \tilde{d}\rangle+\langle\tilde{d}, \tilde{v}-\tilde{a}\rangle=\frac{\alpha_{0}}{\langle v, v\rangle t^{2}+\beta},
$$

and hence

$$
s^{\prime}(t)=\frac{\alpha_{2} t}{t^{2}+\beta_{1}}
$$

with some $\alpha_{2} \in \mathbb{R}, \alpha_{2} \neq 0$. This leads to a contradiction because $\alpha_{2} \neq 0$. Therefore, the case (3.13) does not occur. Interchanging the role of $f$ and $f^{-1}$, the same argument shows that the case (3.14) does not occur.

Next we shall show $\beta=0$ in (3.12). Assume that $\beta \neq 0$. The equation (3.12) shows that for each $r \in \mathbb{R}, f$ maps the intersection of $D$ and hypersurfaces $S_{r}=$ $\{x ;\langle x-a, x-a\rangle+\beta=r\}$ into hypersurfaces $\tilde{S}_{r}=\left\{y ;\langle y-\tilde{a}, y-\tilde{a}\rangle+\tilde{\beta}=\alpha_{0} / r\right\}$. Choose $v \in \mathbb{R}^{n+1}$ with $\langle v, v\rangle \neq 0$ such that the line $x(t)=t v+a(t \in \mathbb{R})$ intersects $D$. In general, $x(t)$ is Lorentz orthogonal to all $S_{r}(r \in \mathbb{R})$ and $f$ is Lorentzian conformal, the image curve $f(x(t))$ of the line $x(t)$ is Lorentz orthogonal to all $\tilde{S}_{r}(r \in \mathbb{R})$ and hence $f(x(t))$ lies in the line $s \tilde{v}+\tilde{a}(s \in \mathbb{R})$ with some $\tilde{v} \in \mathbb{R}^{1, n}$. So there exists a $C^{4}$ function $s(t)$ such that $f(x(t))=s(t) \tilde{v}+\tilde{a}$. Differentiating both sides of the equation by $t$, we have

$$
f^{\prime}(x(t)) v=s^{\prime}(t) \tilde{v}
$$

Then Lorentzian conformality of $f$ implies

$$
s^{\prime}(t)^{2}\langle\tilde{v}, \tilde{v}\rangle=\lambda_{f}(x(t))^{2}\langle v, v\rangle=\frac{\alpha^{2}\langle v, v\rangle}{\left(\langle v, v\rangle t^{2}+\beta\right)^{2}}
$$


and hence

$$
s^{\prime}(t)=\frac{\alpha_{1}}{t^{2}+\beta_{1}},
$$

where $\beta_{1}=\beta /\langle v, v\rangle \neq 0$ and $\alpha_{1}$ is a non-zero constant. Therefore $s(t)$ is inverse tangent function or logarithmic function. In any case, $s(t)$ is a transcendental function. On the other hand, substituting $f(x(t))=s(t) \tilde{v}+\tilde{a}$ and $x(t)=t v+a$ into (3.12), we have

$$
s(t)^{2}\langle\tilde{v}, \tilde{v}\rangle+\tilde{\beta}=\frac{\alpha_{0}}{\langle v, v\rangle t^{2}+\beta},
$$

which shows that $s(t)$ is an algebraic function. This is a contradiction. Therefore $\beta=0$ in (3.12).

Finally we shall show $\langle d, d\rangle=0$ in (3.15). Assume that $\langle d, d\rangle \neq 0$. The equation (3.15) shows that for each $r \in \mathbb{R}, f$ maps the intersection of $D$ and hyperplanes $P_{r}=\{x ;\langle d, x-a\rangle=r\}$ into hyperplanes $\tilde{P}_{r}=\left\{y ;\langle\tilde{d}, y-\tilde{a}\rangle=\alpha_{0} / r\right\}$. Choose $v \in \mathbb{R}^{n+1}$ such that the line $x(t)=t d+v(t \in \mathbb{R})$ intersects $D$. In general, $x(t)$ is Lorentz orthogonal to all $P_{r}(r \in \mathbb{R})$ and $f$ is Lorentzian conformal, the image $f(x(t))$ of the line $x(t)$ is Lorentz orthogonal to all $\tilde{P}_{r}(r \in \mathbb{R})$ and hence $f(x(t))$ lies in the line $s \tilde{d}+\tilde{v}(s \in \mathbb{R})$ with some $\tilde{v} \in \mathbb{R}^{1, n}$. So there exists a $C^{4}$-function $s(t)$ such that $f(x(t))=s(t) \tilde{d}+\tilde{v}$. Differentiating the both sides of the equation by $t$, we have

$$
f^{\prime}(x(t)) d=s^{\prime}(t) \tilde{d}
$$

Since $f$ is Lorentzian conformal,

$$
s^{\prime}(t)^{2}\langle\tilde{d}, \tilde{d}\rangle=\left\langle f^{\prime}(x(t)) d, f^{\prime}(x(t)) d\right\rangle=\lambda_{f}(x(t))^{2}\langle d, d\rangle=\frac{\langle d, d\rangle}{(\langle d, d\rangle t+b)^{2}} .
$$

If $\langle d, d\rangle \neq 0$, this equation implies that $\langle\tilde{d}, \tilde{d}\rangle \neq 0$ and that $s(t)$ is a logarithm function. On the other hand, (3.15) implies that $s(t)$ is a rational function

$$
s(t)\langle\tilde{d}, \tilde{d}\rangle+\tilde{\beta}=\frac{\alpha_{0}}{\langle v, v\rangle t+\beta},
$$

where $\beta=\langle d, v-a\rangle$ and $\tilde{\beta}=\langle\tilde{d}, \tilde{v}-\tilde{a}\rangle$. This is a contradiction. Therefore $\langle d, d\rangle=0$.

Proof of Theorem 1.1. In the following, we shall show that the above cases (3.9), (3.10) and (3.11) corresponds to the cases (a), (b) and (c) of Theorem 1.1.

First we study the case (3.11):

$$
\lambda_{f}(x)=\alpha, \quad \alpha \in \mathbb{R}, \alpha>0 .
$$

By Lemma $3.3, f$ is equal to a similarity

$$
f(x)=\alpha R x+b, \quad\left(R \in \mathrm{O}(1, n), b \in \mathbb{R}^{1, n}\right) .
$$

This is the case (c) of Theorem 1.1.

Next we study the case (3.9):

$$
\lambda_{f}(x)=\frac{\alpha}{\langle x-a, x-a\rangle}, \quad \alpha \in \mathbb{R}, \alpha \neq 0, a \in \mathbb{R}^{1, n} .
$$


If we put $g(x)=x-a$, then $g^{-1}(K(x))=K(x)+a$ and

$$
\begin{aligned}
\lambda_{f \circ g^{-1} \circ K} & =\lambda_{f}(K(x)+a) \lambda_{g^{-1}}(K(x)) \lambda_{K}(x)=\frac{\alpha}{\langle K(x), K(x)\rangle} \cdot 1 \cdot \frac{1}{|\langle x, x\rangle|} \\
& =|\alpha|
\end{aligned}
$$

which implies that $\left(f \circ g^{-1} \circ K\right)(x)$ is equal to a similarity

$$
h(x)=\alpha R x+b, \quad\left(R \in \mathrm{O}(1, n), b \in \mathbb{R}^{1, n}\right) .
$$

Thus we have $\left(f \circ g^{-1} \circ K\right)(y)=h(y)$ and

$$
f(x)=(h \circ K \circ g)(x), \quad x \in D,
$$

because $K^{-1}(x)=K(x)$. This is the case (a) of Theorem 1.1.

Finally we study the case (3.10):

$$
\lambda_{f}(x)=\frac{\alpha}{\langle d, x-a\rangle}, \quad \alpha \in \mathbb{R}, a, d \in \mathbb{R}^{1, n}, \alpha \neq 0, d_{0}=1,\langle d, d\rangle=0 .
$$

Since $\langle d, d\rangle=0$ and $d_{0}=1,\left|\left(d_{1}, \ldots, d_{n}\right)\right|=\left|d_{0}\right|=1$. There exists a matrix $R_{1} \in$ $\mathrm{O}(1, n)$ such that $d=R_{1} v$, where $v=(-1,1,0, \ldots, 0)$. Then $\langle d, x-a\rangle=\left\langle R_{1} v, x-a\right\rangle=$ $\left\langle v, R_{1}^{-1}(x-a)\right\rangle$ holds. Put $S=R_{1}^{-1}$ and define the similarity $g$ as $g(x)=R_{1}^{-1}(x-a)$, so that $\langle d, x-a\rangle=\langle v, g(x)\rangle$. Then

$$
\lambda_{f}\left(g^{-1}(x)\right)=\frac{\alpha}{\left\langle d, g^{-1}(x)-a\right\rangle}=\frac{\alpha}{\left\langle v, g\left(g^{-1}(x)\right)\right\rangle}=\frac{\alpha}{\langle v, x\rangle}=\frac{\alpha}{x_{0}+x_{1}} .
$$

Now considering the mapping $f \circ g^{-1} \circ B^{-1}$ defined on $B(g(D))$, we have

$$
\begin{aligned}
\lambda_{f \circ g^{-1} \circ B^{-1}}(y) & =\lambda_{f}\left(g^{-1}\left(B^{-1}(y)\right)\right) \lambda_{g^{-1}}\left(B^{-1}(y)\right) \lambda_{B^{-1}}(y) \\
& =|\alpha|,
\end{aligned}
$$

which implies that $\left(f \circ g^{-1} \circ B^{-1}\right)(y)$ is equal to a similarity

$$
h(y)=\alpha R y+b, \quad\left(R \in \mathrm{O}(1, n), b \in \mathbb{R}^{1, n}\right) .
$$

Thus we have $\left(f \circ g^{-1} \circ B^{-1}\right)(y)=h(y)$ and

$$
f(x)=(h \circ B \circ g)(x), \quad x \in D .
$$

This is the case (b) of Theorem 1.1.

\section{Transformation which preserves the wave equation}

In this section, we shall give the proof of Theorem 1.2.

By definition, it is easy to see that if $\left(f, \varphi_{f}\right)$ and $\left(g, \varphi_{g}\right)$ are transformations which preserve the wave equation such that the image of $f$ is contained in the domain of $g$, then $\left(g \circ f, \varphi_{f} \cdot\left(\varphi_{g} \circ f\right)\right)$ is also a transformation which preserves the wave equation. We call this transformation $\left(g \circ f, \varphi_{f} \cdot\left(\varphi_{g} \circ f\right)\right)$ the composition of $\left(f, \varphi_{f}\right)$ and $\left(g, \varphi_{g}\right)$. First, we remark that all the transformations in Theorem 1.2 are the composition of the following fundamental transformations: 
Example 4.1 (similarity). The pair of a mapping $f$ and a positive constant function $\varphi$

$$
f(x)=\alpha R x+a, \quad \varphi(x)=C,
$$

where $\alpha \in \mathbb{R}, \alpha>0, R \in \mathrm{O}(1, n), a \in \mathbb{R}^{n+1}$, is a transformation which preserve the wave equation.

Example 4.2 (inversion). The pair of the mapping $K$ and the function $\varphi$

$$
K(x)=\frac{x}{\langle x, x\rangle}, \quad \varphi_{K}(x)=|\langle x, x\rangle|^{\frac{1-n}{2}},
$$

is a transformation which preserve the wave equation defined on each connected component of $\left\{x \in \mathbb{R}^{n+1} ;\langle x, x\rangle \neq 0\right\}$.

Example 4.3 (Bateman transformation). The pair of the mapping $B$ and $\varphi$

$$
\begin{aligned}
B(x) & =\left(\frac{\langle x, x\rangle+1}{2\left(x_{0}+x_{1}\right)}, \frac{\langle x, x\rangle-1}{2\left(x_{0}+x_{1}\right)}, \frac{x_{2}}{x_{0}+x_{1}}, \ldots, \frac{x_{n}}{x_{0}+x_{1}}\right), \\
\varphi_{B}(x) & =\left|x_{0}+x_{1}\right|^{\frac{1-n}{2}}
\end{aligned}
$$

is a transformation which preserve the wave equation defined on each connected component half space of $\left\{x \in \mathbb{R}^{n+1} ; x_{0}+x_{1} \neq 0\right\}$. We call this transformation Bateman transformation.

The following theorem gives a characterization of the transformations which preserve the wave equation.

Theorem 4.1. ([4]) The pair $(f, \varphi)$ is a transformation which preserves the wave equation, if and only if $f=\left(f_{0}, f_{1}, \ldots, f_{n}\right)$ and $\varphi$ satisfy the following equations on $D$ :

$$
\begin{array}{ll}
W \varphi=0, & \\
\varphi W f_{j}-2\left\langle\nabla \varphi, \nabla f_{j}\right\rangle=0, & (j=0,1, \ldots, n) \\
\left\langle\nabla f_{j}, \nabla f_{k}\right\rangle=0, & (j, k=0,1, \ldots, n, j \neq k) \\
\left\langle\nabla f_{j}, \nabla f_{j}\right\rangle=-\left\langle\nabla f_{0}, \nabla f_{0}\right\rangle, & (j=1, \ldots, n)
\end{array}
$$

where $\nabla f_{j}=\left(\frac{\partial f_{j}}{\partial x_{0}}, \frac{\partial f_{j}}{\partial x_{1}}, \ldots, \frac{\partial f_{j}}{\partial x_{n}}\right), j=0,1, \ldots, n$.

Proof. Since [4] is written in Japanese, we write the proof here. For any $C^{2}$-function $u$,

$$
\begin{aligned}
& W(\varphi \cdot(u \circ f))=(W \varphi) \cdot(u \circ f) \\
& \quad+\sum_{j=0}^{n}\left(\varphi \cdot W f_{j}-2\left\langle\nabla \varphi, \nabla f_{j}\right\rangle\right) \cdot\left(\frac{\partial u}{\partial y_{j}} \circ f\right)-\sum_{j, k=0}^{n} \varphi \cdot\left\langle\nabla f_{j}, \nabla f_{k}\right\rangle \cdot\left(\frac{\partial^{2} u}{\partial y_{j} \partial y_{k}} \circ f\right)
\end{aligned}
$$


holds by the chain rule. Assume that $(f, \varphi)$ is a transformation which preserves the wave equation. First we take the solution $u=1$. Since $\frac{\partial u}{\partial y_{j}}=\frac{\partial^{2} u}{\partial y_{j} \partial y_{k}}=0(0 \leqq j, k \leqq$ $n)$, we have (4.1):

$$
W \varphi=0 .
$$

Next take the solution $u(y)=y_{l}(0 \leqq l \leqq n)$. Since $\frac{\partial u}{\partial y_{j}}=\delta_{j l}, \frac{\partial^{2} u}{\partial y_{j} \partial y_{k}}=0(0 \leqq j, k \leqq$ $n)$, we have (4.2):

$$
\varphi W f_{j}-2\left\langle\nabla \varphi, \nabla f_{j}\right\rangle=0 .
$$

Take the solution $u(y)=y_{i} y_{l}(0 \leqq i, l \leqq n, i \neq l)$. Since $\frac{\partial^{2} u}{\partial y_{j} \partial y_{k}}=\delta_{i j} \delta_{k l}(0 \leqq j, k \leqq$ $n)$, we have (4.3):

$$
\left\langle\nabla f_{i}, \nabla f_{l}\right\rangle=0 \quad(0 \leqq i, l \leqq n, i \neq l) .
$$

Finally take the solution $u(y)=y_{0}^{2}+y_{j}^{2}(0 \leqq j \leqq n)$. Since $\frac{\partial^{2} u}{\partial y_{0}^{2}}=\frac{\partial^{2} u}{\partial y_{j}^{2}}=2, \frac{\partial^{2} u}{\partial y_{i}^{2}}=0$ $(1 \leqq i \leqq n, i \neq j)$, we have (4.4):

$$
\left\langle\nabla f_{0}, \nabla f_{0}\right\rangle+\left\langle\nabla f_{j}, \nabla f_{j}\right\rangle=0 .
$$

Conversely assume that $f$ and $\varphi$ satisfy (4.1),..,(4.4). Substituting (4.1), (4.2) and (4.3) into (4.5), we have

$$
W(\varphi \cdot(u \circ f))=-\varphi \sum_{j=0}^{n}\left\langle\nabla f_{j}, \nabla f_{j}\right\rangle \cdot\left(\frac{\partial^{2} u}{\partial y_{j}^{2}} \circ f\right) .
$$

Putting $\Lambda=\left\langle\nabla f_{0}, \nabla f_{0}\right\rangle,(4.4)$ gives

$$
W(\varphi \cdot(u \circ f))(x)=-\varphi(x) \Lambda(x)((W u) \circ f)(x) .
$$

This shows that $W u=0$ implies $W(\varphi \cdot(u \circ f))=0$. Therefore $(\varphi, f)$ is a transformation which preserve the wave equation.

Corollary 4.1. Let $n \geqq 2$. If $(f, \varphi)$ is a transformation which preserves the wave equation defined on $D$, then $f$ is Lorentzian conformal on $D$ such that $\lambda(x)^{2}=$ $\left\langle\nabla f_{0}, \nabla f_{0}\right\rangle$.

Proof. Putting $\Lambda=\left\langle\nabla f_{0}, \nabla f_{0}\right\rangle,(4.3)$ and (4.4) is equivalent to

$$
f^{\prime}(x) J^{t} f^{\prime}(x)=\Lambda(x) J
$$

Since $f$ is non-degenerate, $\Lambda \neq 0$. Then ${ }^{t} f^{\prime}(x)^{-1} J f^{\prime}(x)^{-1}=\Lambda(x)^{-1} J$ and hence ${ }^{t} f^{\prime}(x) J f^{\prime}(x)=\Lambda(x) J$, which is equivalent to $\left\langle f^{\prime}(x) u, f^{\prime}(x) v\right\rangle=\Lambda(x)\langle u, v\rangle$ for all $u, v \in \mathbb{R}^{1, n}$. Since $f^{\prime}(x)$ is invertible, the quadratic form $\left\langle f^{\prime}(x) u, f^{\prime}(x) u\right\rangle$ has the same signature as $\langle u, u\rangle$. Therefore $\Lambda(x)>0$ for all $x \in D$, because $n \geqq 2>1$. Thus $f$ is Lorentzian conformal on $D$.

Next lemma shows that $\varphi$ is uniquely determined by $f$ except a constant multiple. 
Lemma 4.1. If $\left(f, \varphi_{1}\right)$ and $\left(f, \varphi_{2}\right)$ are two transformation which preserve the wave equation with same mapping $f$ on a domain. Then $\varphi_{2}$ is a constant multiple of $\varphi_{1}$.

Proof. By (4.2), we have

$$
W f_{j}=2\left\langle\nabla \log \varphi_{1}, \nabla f_{j}\right\rangle, \quad W f_{j}=2\left\langle\nabla \log \varphi_{2}, \nabla f_{j}\right\rangle, \quad(j=0,1, \ldots, n) .
$$

and then

$$
\left\langle\nabla f_{j}, \nabla \log \frac{\varphi_{2}}{\varphi_{1}}\right\rangle=\left\langle\nabla f_{j}, \nabla \log \varphi_{2}-\nabla \log \varphi_{1}\right\rangle=0, \quad(j=0,1, \ldots, n) .
$$

Hence $f^{\prime}(x)$ maps the vector $\nabla \log \frac{\varphi_{2}}{\varphi_{1}}(x)$ to 0 . Since the matrix $f^{\prime}(x)$ is non-degenerate, $\nabla \log \frac{\varphi_{2}}{\varphi_{1}}(x)=0$ for all $x \in D$. Therefore, $\varphi_{2}$ is a constant multiple of $\varphi_{1}$.

Proof of Theorem 1.2. By Corollary 4.1, $f$ is Lorentzian conformal on $D$. Then by Theorem 1.1, $f$ has the form (a), (b), or (c).

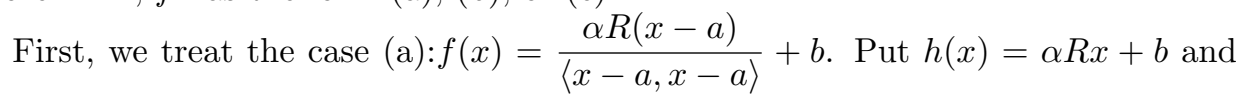
$g(x)=x-a$. Then $f(x)=(h \circ K \circ g)(x)$. Since $(h, 1)$ and $(g, 1)$ are transformations which preserve the wave equation, Lemma 4.1 yields that $\varphi$ is a constant multiple of

$$
1 \cdot\left(\varphi_{K} \circ g\right) \cdot(1 \circ K \circ g)=|\langle x-a, x-a\rangle|^{\frac{1-n}{2}} .
$$

Therefore $\varphi(x)=C|\langle x-a, x-a\rangle|^{\frac{1-n}{2}}$ with $C>0$.

Next we treat the case (b): $f(x)=\alpha R B(S(x-a))+b$. Put $h(x)=\alpha R x+b$ and $g(x)=S(x-a)$. Then $f(x)=(h \circ B \circ g)(x)$. Since $(h, 1)$ and $(g, 1)$ are transformations which preserve the wave equation, $\varphi$ is a constant multiple of

$$
1 \cdot\left(\varphi_{B} \circ g\right) \cdot(1 \circ B \circ g)=|\langle v, S(x-a)\rangle|^{\frac{1-n}{2}}
$$

by Lemma 4.1. Therefore $\varphi(x)=C|\langle v, S(x-a)\rangle|^{\frac{1-n}{2}}$ with $C>0$.

Finally, $f(x)=\alpha R x+b$ is the similarity. So $\varphi$ is a positive constant.

Acknowledgements: The author would like to express his gratitude to the referee for his valuable comments.

\section{References}

[1] M. Berger, Geometry, Springer-Verlag, 1986.

[2] P. Hillion, The Cournat-Hilbert solutions of the wave equation, J. Math. Phys. 33(8), 1992, 2749-2753.

[3] J.G. Ratcliffe, Foundations of hyperbolic manifolds, 2nd edition, Springer-Verlag, 2006.

[4] T. Sugimoto, On Kelvin type transfomations associated with the wave equation (in Japanese), Master thesis (Nagoya University), 1997. 